\title{
Association between Polymorphisms of Alpha-Adducin Gene and Essential Hypertension in Chinese Population
}

\author{
Li-na Zhang, ${ }^{1}$ Lin-dan Ji, ${ }^{2,3}$ Li-juan Fei, ${ }^{1}$ Fang Yuan, ${ }^{1}$ Yue-miao Zhang, ${ }^{1}$ and Jin Xu ${ }^{1,3}$ \\ ${ }^{1}$ Department of Preventive Medicine, School of Medicine, Ningbo University, Ningbo 315211, China \\ ${ }^{2}$ Department of Biochemistry, School of Medicine, Ningbo University, Ningbo 315211, China \\ ${ }^{3}$ State Key Laboratory of Genetic Resources and Evolution, Kunming Institute of Zoology, Chinese Academy of Sciences, \\ Kunming 650223, China
}

Correspondence should be addressed to Jin Xu; xujin1@nbu.edu.cn

Received 6 September 2012; Revised 24 October 2012; Accepted 25 October 2012

Academic Editor: Joseph Fomusi Ndisang

Copyright (C) 2013 Li-na Zhang et al. This is an open access article distributed under the Creative Commons Attribution License, which permits unrestricted use, distribution, and reproduction in any medium, provided the original work is properly cited.

\begin{abstract}
The association between polymorphisms of $\alpha$-adducin (ADD1) gene and essential hypertension is still unclear. Thus, we carried out a case-control study and an interaction analysis to test whether $A D D 1$ is a common candidate gene for hypertension in the Chinese population. Blood samples and information including body mass index (BMI), smoking habit, and alcohol abuse were collected. Meanwhile, total cholesterol, high density lipoprotein, triglyceride were measured by automatic biochemistry analyzer. All 6 tag single nucleotide polymorphisms (tagSNPs) within ADD1 gene were genotyped by SNPstream genotyping system. Multifactor dimensionality reduction (MDR) was used to identify the interactions among the SNPs and the non-genetic factors. Results showed that plasma triglyceride, total cholesterol, and BMI were significantly higher in the hypertensive group than in the control group. Result from genotyping indicated that rs 4963 was significantly associated with essential hypertension. After stratification by gender, rs4963 was associated with essential hypertension only in males. MDR analysis indicated that interaction among BMI, rs4963, and rs16843452 were involved in susceptibility of hypertension. The present study indicated that rs4963 within ADD1 gene was associated with essential hypertension in Chinese population, which might be related to altered exonic splicing and disrupted gene regulation.
\end{abstract}

\section{Introduction}

Adducin (ADD) is a heterodimeric cytoskeleton protein consisting of an $\alpha$-subunit with either a $\beta$ - or a $\gamma$-subunit [1], and they are encoded by three different genes $A D D 1$, $A D D 2$, and $A D D 3$, respectively. The $\alpha$-subunit is known to increase renal sodium reabsorption and may be involved in the pathophysiology of essential hypertension [2]. Therefore, it is considered as a major candidate gene for essential hypertension. Not surprisingly, a number of studies have investigated the association of $\alpha$-adducin gene (ADD1) polymorphisms with essential hypertension in the past two decades.

Human ADD1 gene is located on chromosome 4p16.3 and comprises 16 exons. One well-studied polymorphism in $A D D 1$ gene is a substitution of Gly for Trp at amino acid residue 460 (G460W, rs4961), which was first described by
Cusi et al. [3]. ADD1 W460W was found to be associated with a form of low renin salt-sensitive hypertension in Caucasians, with reduced response to pressure natriuretic function and with increased proximal sodium reabsorption $[4,5]$. Therefore, although a great number of similar studies have been conducted, the results are often inconsistent.

To convince the association of this polymorphism with essential hypertension, several meta-analyses were recently published [6-10]. Most of these analyses failed to provide evidence for the genetic association of $A D D 1$ G460W polymorphism with essential hypertension in global population; but it is suggested that the $\mathrm{W}$ carrier might increase the risk of essential hypertension in Han Chinese population [7]. However, most studies focus on ADD1 G460W polymorphism, and whether other single nucleotide polymorphisms (SNPs) in $A D D 1$ gene were associated with essential hypertension is not clear. Therefore, we carried out a case-control study 
and an interaction analysis to verify whether $A D D 1$ gene is associated with hypertension in the Chinese population.

\section{Materials and Methods}

2.1. Population for Case-Control Study. The participants were chosen from our established community-based epidemiology study of common diseases. We have collected more than 10,000 health records. Subsequently, participants who fulfilled the following criteria were put into our database: 30 to 75 years old, Han Chinese, living in Ningbo City (East Coast of China) for at least three generations and do not have a migration history. The hypertensive subjects and control subjects were matched for age and sex. In addition, only subjects who do not have other cardiovascular disease or major chronic illnesses according to their health records were included in the control group. Lastly, 905 essential hypertension cases and 905 healthy controls, including 392 males and 513 females in each group, were involved in the following studies.

2.2. Blood Pressure and Clinical Parameters. Blood pressure measurements were conducted in the morning after the participant had been in the sitting position for $10 \mathrm{~min}$ utes. Three BP measurements were obtained at 5-minute intervals using standard mercury sphygmomanometer, and the average of last two measurements was taken as the BP for that participant. Hypertension in this study is defined as a sitting systolic blood pressure $(\mathrm{SBP}) \geq 140 \mathrm{mmHg}$ and/or a diastolic blood pressure (DBP) $\geq 90 \mathrm{mmHg}$, or selfreported use of antihypertension medication (also confirmed by health records). Patients with secondary hypertension were excluded. Normal blood pressure is defined with SBP $\leq 120 \mathrm{mmHg}$ and $\mathrm{DBP} \leq 80 \mathrm{mmHg}$.

Blood samples were collected with informed consent. Subsequently, total cholesterol (TC), high density lipoprotein (HDL), and triglyceride (TG) were measured from these blood samples by Hitachi automatic biochemistry analyzer 7100. Clinical information including body mass index (BMI), and weekly alcohol and cigarettes consumption were also obtained. In this study, who drank $\geq 70 \mathrm{~g}$ alcohol per week for more than 1 year were defined as individuals with alcohol abuse. Moreover, who smoked $\geq 70$ cigarettes per week for more than 1 year were defined as individuals with smoking habit. The protocol of this study was reviewed and approved by the Ethics Committees of Ningbo University.

2.3. SNP Genotyping. All six tagSNPs were retrieved from HapMap (http://hapmap.ncbi.nlm.nih.gov/), with tagger pairwise method in CHB: $R$ Square cut off $=0.8$ and MAF cut off $=0.1$. Genomic DNA was obtained from the whole blood using standard phenol/chloroform extraction. Genotyping was performed using the GenomeLab SNPstream Genotyping System (Beckman Coulter Inc.) according to the manufacturer's protocol [11].

2.4. Statistical Analysis. Continuous variables are presented as the mean $\pm \mathrm{SD}$ and analyzed by $t$-test between two
TABLE 1: Baseline characters of the investigated participants.

\begin{tabular}{lccc}
\hline Variables & Hypertensive & Control & $P$ value \\
\hline Number & 905 & 905 & N/A \\
Sex (male) (\%) & 43.3 & 43.3 & N/A \\
Age $(y)$ & $56.91 \pm 7.37$ & $56.60 \pm 7.51$ & $P=0.38$ \\
TG $(\mathrm{mM})$ & $2.02 \pm 1.68$ & $1.63 \pm 1.12$ & $P<0.01$ \\
HDL $(\mathrm{mM})$ & $1.41 \pm 0.35$ & $1.41 \pm 0.32$ & $P=0.72$ \\
TC $(\mathrm{mM})$ & $5.34 \pm 1.00$ & $5.17 \pm 0.93$ & $P<0.01$ \\
BMI $\left(\mathrm{Kg} / \mathrm{m}^{2}\right)$ & $24.65 \pm 3.24$ & $23.21 \pm 2.86$ & $P<0.01$ \\
Regular smoking & 173 & 147 & $P=0.11$ \\
Regular alcohol drinking & 152 & 148 & $P=0.80$ \\
\hline
\end{tabular}

TG: triglyceride; HDL: high density lipoprotein; TC: total cholesterol; BMI: body mass index.

groups. Statistical analyses of allele and genotype frequencies between hypertensive cases and healthy controls and between different sex groups were performed by chi-squared test (SPSS 16.0, SPSS Inc.). Hardy-Weinberg equilibrium (HWE) was tested by the software PEDSTATS V0.6.8 (http://www.sph.umich.edu/csg/abecasis/). Linkage equilibrium (LD) blocks were defined based on the confidence intervals method [12] and the most likely haplotypes within each block for individuals were inferred using the Haploview software [13]. Multifactor dimensionality reduction (MDR) was used to identify and characterize interactions among the SNPs and the nongenetic factors, including BMI, serum HDL, TC, and TG level, as well as percentage of smoking and alcohol abuse [14]. The software used for MDR is distributed in a JAVA platform with a graphical user interface and is freely available (http://www.epistasis.org/mdr.html).

All tests were two sided, and $P$ values less than 0.05 were considered statistically significant.

\section{Results}

The baseline characteristics of our study population are summarized in Table 1. Age and sex distribution, HDL, smoking percentage, and percentage of alcohol abuse showed no difference between hypertensive and control groups. However, TG, TC, and BMI were significantly higher in the hypertensive group than in the control group $(P<0.05)$.

Genotype distributions of six tagSNPs within $A D D 1$ gene were shown in Table 2. None of them deviated from the HWE $(P>0.05)$. Only rs4963 was significantly associated with essential hypertension $(P=0.02$, odds ratio $(\mathrm{OR})=0.85$, $95 \%$ confident interval $(\mathrm{CI})=0.75-0.97)$. After stratification by gender, rs4963 was associated with essential hypertension only in males, and the other 5 SNPs were still negative (Table 3). We further performed haplotype analysis within LD blocks of $A D D 1$. Only one LD block and three common haplotypes within the LD block (including rs16843452 and rs12503220) were identified (Figure 1). Of these three haplotypes, no haplotype was significantly associated with hypertension (Table 4).

Finally, MDR was used to analyze the interaction among different SNPs and nongenetic risk factors for hypertension. 
TABLE 2: Association analysis of 6 tagSNPs.

\begin{tabular}{|c|c|c|c|c|c|c|c|}
\hline SNP & Group & & Genotype & & $P$ value & OR & $95 \% \mathrm{CI}$ \\
\hline \multirow{2}{*}{ rs16843452 } & Hypertensive & 243 (CC) & $462(\mathrm{CT})$ & $195(\mathrm{TT})$ & \multirow{2}{*}{0.89} & \multirow{2}{*}{1.01} & \multirow{2}{*}{$0.89 \sim 1.15$} \\
\hline & Control & 243 (CC) & $461(\mathrm{CT})$ & 199 (TT) & & & \\
\hline \multirow{2}{*}{ rs 12503220} & Hypertensive & 11 (AA) & $253(\mathrm{AG})$ & 636 (GG) & \multirow{2}{*}{0.26} & \multirow{2}{*}{0.90} & \multirow{2}{*}{$0.75 \sim 1.08$} \\
\hline & Control & $22(\mathrm{AA})$ & $257(\mathrm{AG})$ & $624(\mathrm{GG})$ & & & \\
\hline \multirow{2}{*}{ rs4963 } & Hypertensive & 192 (CC) & 432 (CG) & 278 (GG) & \multirow{2}{*}{0.02} & \multirow{2}{*}{0.85} & \multirow{2}{*}{$0.75 \sim 0.97$} \\
\hline & Control & $224(\mathrm{CC})$ & 441 (CG) & 239 (GG) & & & \\
\hline \multirow{2}{*}{ rs3755885 } & Hypertensive & $62(\mathrm{CC})$ & 379 (CG) & 461 (GG) & \multirow{2}{*}{0.24} & \multirow{2}{*}{0.92} & \multirow{2}{*}{$0.79 \sim 1.06$} \\
\hline & Control & 67 (CC) & 402 (CG) & 435 (GG) & & & \\
\hline \multirow{2}{*}{ rs2071694 } & Hypertensive & 447 (CC) & 374 (CG) & 82 (GG) & \multirow{2}{*}{0.81} & \multirow{2}{*}{0.98} & \multirow{2}{*}{$0.85 \sim 1.13$} \\
\hline & Control & 455 (CC) & 366 (CG) & 83 (GG) & & & \\
\hline \multirow{2}{*}{ rs3775067 } & Hypertensive & 318 (AA) & $435(\mathrm{AG})$ & $148(\mathrm{GG})$ & \multirow{2}{*}{0.27} & \multirow{2}{*}{1.08} & \multirow{2}{*}{$0.94 \sim 1.23$} \\
\hline & Control & 287 (AA) & $469(\mathrm{AG})$ & 149 (GG) & & & \\
\hline
\end{tabular}

OR: odds ratio; CI: confidence interval.

TABLE 3: Association analysis of 6 tagSNPs in men and women.

\begin{tabular}{lcccc}
\hline SNP & Group & $P$ value & OR & $95 \%$ CI \\
\hline \multirow{2}{*}{ rs16843452 } & Male & 0.51 & 0.94 & $0.77 \sim 1.14$ \\
& Female & 0.45 & 1.07 & $0.90 \sim 1.27$ \\
rs12503220 & Male & 0.31 & 0.87 & $0.66 \sim 1.14$ \\
& Female & 0.53 & 0.93 & $0.73 \sim 1.17$ \\
rs4963 & Male & 0.02 & 0.79 & $0.65 \sim 0.97$ \\
& Female & 0.25 & 0.90 & $0.76 \sim 1.07$ \\
rs3755885 & Male & 0.80 & 1.03 & $0.83 \sim 1.28$ \\
& Female & 0.08 & 0.84 & $0.69 \sim 1.02$ \\
rs2071694 & Male & 0.28 & 0.89 & $0.72 \sim 1.10$ \\
& Female & 0.53 & 1.06 & $0.88 \sim 1.29$ \\
rs3775067 & Male & 0.21 & 1.14 & $0.93 \sim 1.40$ \\
& Female & 0.71 & 1.03 & $0.87 \sim 1.23$ \\
\hline
\end{tabular}

OR: odds ratio; CI: confidence interval.

TABLE 4: Association analysis of haplotypes with hypertension.

\begin{tabular}{lccc}
\hline $\begin{array}{l}\text { Haplotype } \\
\text { sequence }\end{array}$ & $\begin{array}{l}\text { Haplotype } \\
\text { frequency }\end{array}$ & Case/control ratio & $P$ value \\
\hline TG & 0.472 & $0.473,0.470$ & 0.840 \\
CG & 0.368 & $0.360,0.377$ & 0.274 \\
CA & 0.157 & $0.165,0.149$ & 0.208 \\
\hline
\end{tabular}

After input the genotypes of 6 SNPs together with information about TG, TC, HDL, BMI, smoking, and alcohol abuse, the software outputs the best model for "BMI, rs4963, rs16843452" with 10/10 crossvalidation consistency (Table $5)$.

\section{Discussion}

Among the three adducin genes, $A D D 1$ has received more attention than the other two due to its association with several diseases, such as hyperlipidemia [15], renal disease [16], and coronary heart disease [17]. Series of studies in humans reveal that mutation of $A D D 1$ gene may lead to the

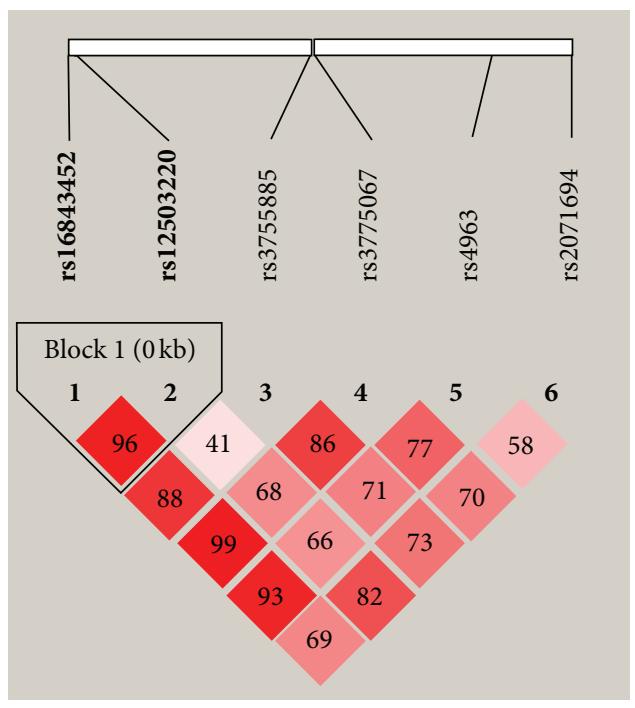

FIgure 1: The LD block of 6 tagSNPs within $A D D 1$ gene. Pairwise linkage disequilibrium (LD) coefficients $D^{\prime} \times 100$ are shown in each cell. Only one LD block (including rs16843452 and rs12503220) is identified by confidence intervals method.

stimulation of the sodium and potassium-adenosine triphosphatase (ATPase) activity in renal tubular cells, increased renal sodium reabsorption, and subsequently hypertension $[18,19]$.

Since hypertension is considered polygenic, resulting from the interaction of several genes and together with environmental factors, a single SNP has weak effects on the phenotype. A family study from van Rijin et al. [20] showed that $A D D 1$ G460W polymorphism was able to explain only a very minute proportion of the heritability of BP traits. Therefore, it is necessary to explore whether there are any other SNPs within ADD1 gene that are also associated with essential hypertension. In order to answer this question, we carried out a case-control study in the Chinese population. The result showed that rs4963 was associated with essential hypertension, especially in males. One possible reason for 
TABLE 5: MDR analysis of gene-environment interaction.

\begin{tabular}{lccccc}
\hline Best model & Testing accuracy & Testing sensitivity & Testing odds ratio & Testing $\chi^{2}$ & Crossvalidation consistency \\
\hline BMI & 0.59 & 0.43 & $2.26(95 \%$ CI: $1.20-4.27)$ & $6.50(P=0.010)$ & $10 / 10$ \\
BMI, rs4963, rs16843452 & 0.61 & 0.54 & $2.42(95 \%$ CI: $1.33-4.44)$ & $8.40(P=0.004)$ & $10 / 10$ \\
BMI, TG & 0.58 & 0.57 & $1.98(95 \%$ CI: $1.10-3.58)$ & $5.18(P=0.023)$ & $7 / 10$ \\
\hline
\end{tabular}

this result might be that men have higher TG (1.93 versus $1.69 \mathrm{mM}$ ) and TC (5.39 versus $5.08 \mathrm{mM}$ ) level than women.

Out of 1,113 SNPs associated with $A D D 1$ gene [21], 9 are identified to be nonsynonymous by functional singlenucleotide polymorphism (F-SNP) database [22]. rs4963 is one of them, and the amino acid change found is from Serine to Cysteine. The G allele of rs4963 has deleterious effect by disrupting the activity of exonic splicing and thus disrupt proper gene regulation [23]. In current study, G allele of rs4963 increases the susceptibility of hypertension, which might be associated with altered protein structure and function.

Moreover, environmental factors and individual's biological characteristics, including excess dietary sodium intake, alcohol abuse, smoking, obesity, stress, BMI, TC, and TG, cannot be neglected [24, 25]. We also found interesting interactions between genetic factors and nongenetic risk factors. High BMI and serum TG level were already confirmed to be risk factors for hypertension [26]. The MDR analysis in this study demonstrated that these important risk factors interacted with the genetic factor. Thus, the present interaction analysis gave a little more information than the single genetic study.

In conclusion, the present study indicated that rs4963 within $A D D 1$ gene was associated with essential hypertension in Chinese population, which might be related to altered exonic splicing and disrupted gene regulation. Regulation of sodium transport is such a complex process that at least 189 genes are involved according to gene ontology (GO: 0002028). ADD1 is only a common member of this system, and polymorphisms within this gene may play a tiny role. Other genes involved in sodium transport regulation system need to be studied. In addition, in our interaction analysis, a significant interaction was found between genetic and nongenetic factors, demonstrating that genetic study alone is not a sufficient indicator for hypertension. To decipher causal factors leading to the development and the pathogenesis of hypertension, future work will require analysis of geneenvironmental interaction.

\section{Authors' Contribution}

These authors contributed equally to this work.

\section{Acknowledgments}

This study was financially supported by Ningbo Natural Science Foundation (2011A610037, 2012A610237), China Postdoctoral Science Foundation (20100481399), and the K. C. Wong Magna Fund in Ningbo University. The authors acknowledge Dr. Ali J. Marian and Dr. Ju-Yun Tsai for providing important suggestions.

\section{References}

[1] Y. Matsuoka, X. Li, and V. Bennett, "Adducin: structure, function and regulation," Cellular and Molecular Life Sciences, vol. 57, no. 6, pp. 884-895, 2000.

[2] C. A. Hughes and V. Bennett, "Adducin: a physical model with implications for function in assembly of spectrin-actin complexes," Journal of Biological Chemistry, vol. 270, no. 32, pp. 18990-18996, 1995.

[3] D. Cusi, C. Barlassina, T. Azzani et al., "Polymorphisms of $\alpha$ adducin and salt sensitivity in patients with essential hypertension," The Lancet, vol. 349, no. 9062, pp. 1353-1357, 1997.

[4] P. Manunta, M. Burnier, M. D’Amico et al., "Adducin polymorphism affects renal proximal tubule reabsorption in hypertension," Hypertension, vol. 33, no. 2, pp. 694-697, 1999.

[5] P. Manunta, D. Cusi, C. Barlassina et al., " $\alpha$-adducin polymorphisms and renal sodium handling in essential hypertensive patients," Kidney International, vol. 53, no. 6, pp. 1471-1478, 1998.

[6] K. Liu, J. Liu, Y. Huang et al., "Alpha-adducin Gly460Trp polymorphism and hypertension risk: a meta-analysis of 22 studies including 14303 cases and 15961 controls," PLOS ONE, vol. 5, no. 9, Article ID e13057, 2010.

[7] K. Liu, Y. Liu, J. Liu et al., " $\alpha$-adducin Gly460Trp polymorphism and essential hypertension risk in Chinese: a meta-analysis," Hypertension Research, vol. 34, no. 3, pp. 389-399, 2011.

[8] W. Niu and Y. Qi, "Association of $\alpha$-adducin and G-protein $\beta 3$ genetic polymorphisms with hypertension: a meta-analysis of Chinese populations," PLOS ONE, vol. 6, no. 2, Article ID e17052, 2011.

[9] W. Q. Niu, Y. Zhang, K. D. Ji, P. J. Gao, and D. L. Zhu, "Lack of association between $\alpha$-adducin G460W polymorphism and hypertension: evidence from a case-control study and a metaanalysis," Journal of Human Hypertension, vol. 24, no. 7, pp. 467-474, 2010.

[10] R. Wang, B. Zhong, Y. Liu, and C. Wang, "Association between $\alpha$-adducin gene polymorphism (Gly460Trp) and genetic predisposition to salt sensitivity: a meta-analysis," Journal of Applied Genetics, vol. 51, no. 1, pp. 87-94, 2010.

[11] P. A. Bell, S. Chaturvedi, C. A. Gelfand et al., "SNPstream:?: UHT: ultra-high throughput SNP genotyping for pharmacogenomics and drug discovery," BioTechniques, vol. 32, supplement, pp. S70-S77, 2002.

[12] S. B. Gabriel, S. F. Schaffner, H. Nguyen et al., "The structure of haplotype blocks in the human genome," Science, vol. 296, no. 5576, pp. 2225-2229, 2002.

[13] J. C. Barrett, B. Fry, J. Maller, and M. J. Daly, "Haploview: analysis and visualization of LD and haplotype maps," Bioinformatics, vol. 21, no. 2, pp. 263-265, 2005. 
[14] A. A. Motsinger and M. D. Ritchie, "The effect of reduction in cross-validation intervals on the performance of multifactor dimensionality reduction," Genetic Epidemiology, vol. 30, no. 6, pp. 546-555, 2006.

[15] E. Beeks, R. G. J. H. Janssen, A. A. Kroon et al., "Association between the $\alpha$-adducin Gly460Trp polymorphism and systolic blood pressure in familial combined hyperlipidemia," American Journal of Hypertension, vol. 14, no. 12, pp. 1185-1190, 2001.

[16] C. Zoccali, "ACE and $\alpha$-adducin genotypes and renal disease progression," Nephrology Dialysis Transplantation, vol. 15, supplement 6, pp. 69-71, 2000.

[17] M. H. Zafarmand, Y. T. van der Schouw, D. E. Grobbee, P. W. De Leeuw, and M. L. Bots, “ $\alpha$-adducin Gly460Trp variant increases the risk of stroke in hypertensive Dutch women," Hypertension, vol. 51, no. 6, pp. 1665-1670, 2008.

[18] A. M. Castejon, A. B. Alfieri, I. S. Hoffmann, A. Rathinavelu, and L. X. Cubeddu, "Alpha-adducin polymorphism, salt sensitivity, nitric oxide excretion, and cardiovascular risk factors in normotensive hispanics," American Journal of Hypertension, vol. 16, no. 12, pp. 1018-1024, 2003.

[19] J. A. Staessen and G. Bianchi, "Adducin and hypertension," Pharmacogenomics, vol. 6, no. 7, pp. 665-669, 2005.

[20] M. J. E. van Rijn, A. F. Schut, Y. S. Aulchenko et al., "Heritability of blood pressure traits and the genetic contribution to blood pressure variance explained by four blood-pressure-related genes," Journal of Hypertension, vol. 25, no. 3, pp. 565-570, 2007.

[21] E. W. Sayers, T. Barrett, D. A. Benson et al., "Database resources of the national center for biotechnology information," Nucleic Acids Research, vol. 40, pp. D13-D25, 2012.

[22] P. H. Lee and H. Shatkay, "An integrative scoring system for ranking SNPs by their potential deleterious effects," Bioinformatics, vol. 25, no. 8, pp. 1048-1055, 2009.

[23] A. Kundu and A. Anand, "Computational study of ADDlgene polymorphism associated with hypertension," Cell Biochemistry and Biophysics. In press.

[24] P. A. Doris, "Hypertension genetics, single nucleotide polymorphisms, and the common disease: common variant hypothesis," Hypertension, vol. 39, no. 2, pp. 323-331, 2002.

[25] A. Lev-Ran and M. Porta, "Salt and hypertension: a phylogenetic perspective," Diabetes/Metabolism Research and Reviews, vol. 21, no. 2, pp. 118-131, 2005.

[26] V. Bhan, R. T. Yan, L. A. Leiter et al., "Relation between obesity and the attainment of optimal blood pressure and lipid targets in high vascular risk outpatients," American Journal of Cardiology, vol. 106, no. 9, pp. 1270-1276, 2010. 


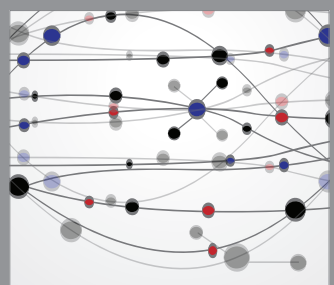

The Scientific World Journal
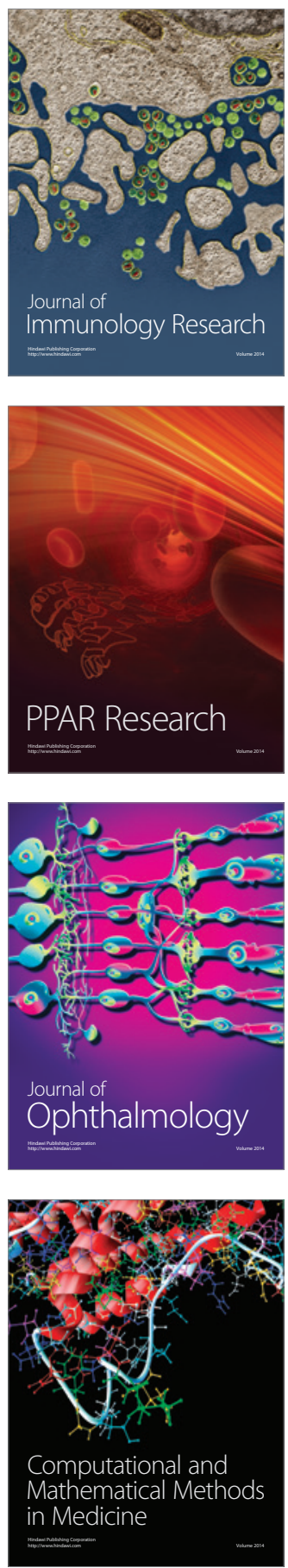

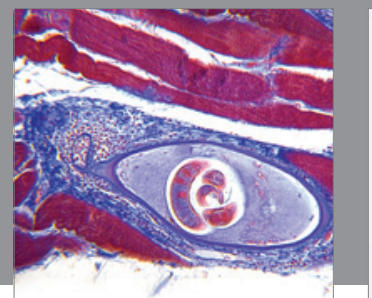

Gastroenterology

Research and Practice
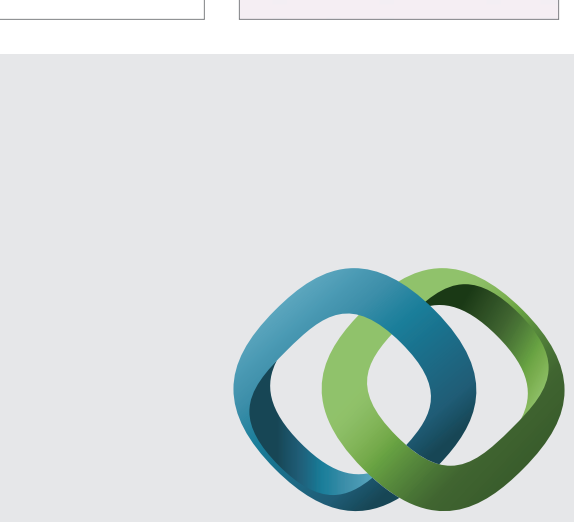

\section{Hindawi}

Submit your manuscripts at

http://www.hindawi.com
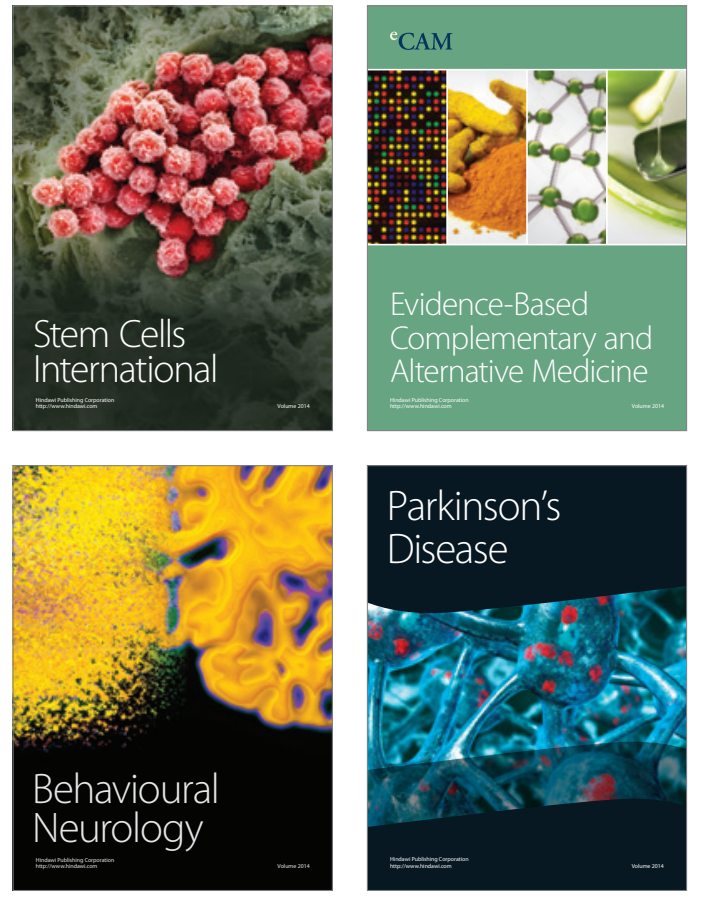
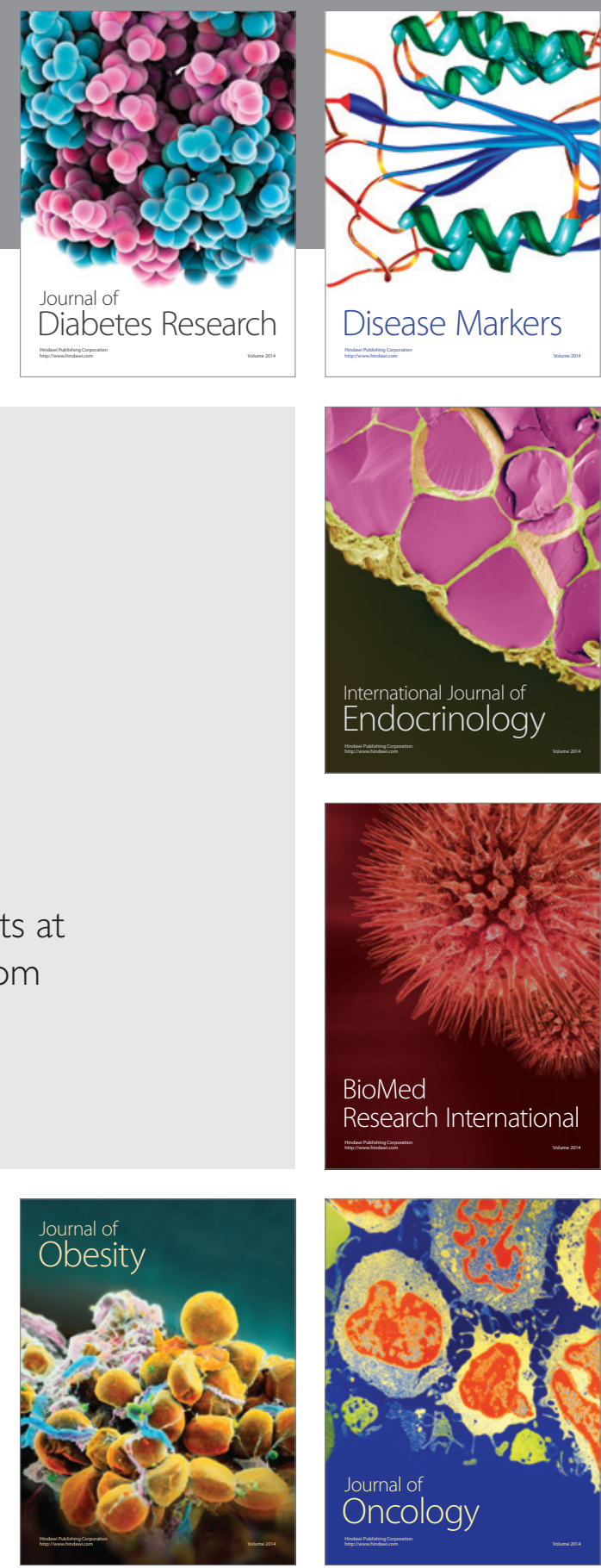

Disease Markers
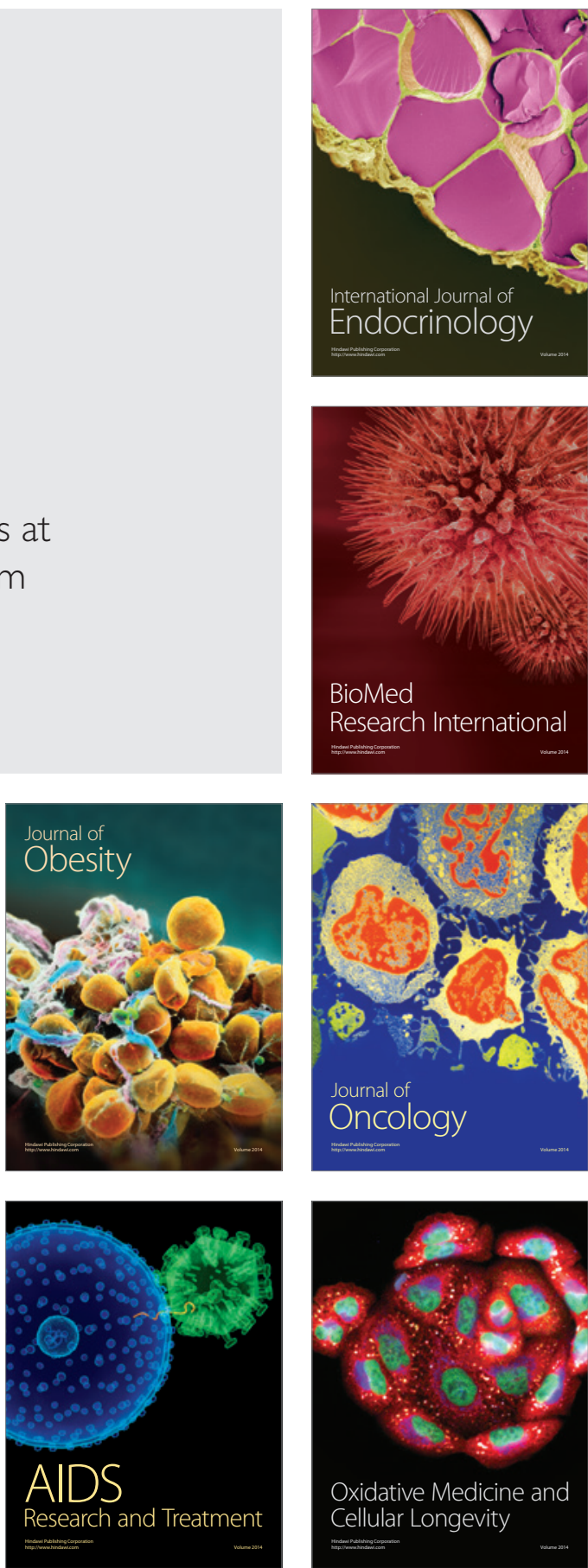